\title{
Translation: A Key Component of a Hundred-Year Project
}

\author{
Tsondue Samphel, Dadul Namgyal ${ }^{*}$, Dawa Tsering Drongbu and Karma Tenzin Khangsar \\ Center for Contemplative Science and Compassion-Based Ethics, Emory University, Atlanta, GA, United States
}

The Emory-Tibet Science Initiative (ETSI) has embarked on a historic endeavor of introducing a systematic and sustainable science education program within the traditional Tibetan monastic institutions. His Holiness the Dalai Lama, who conceived and supports this initiative, calls it a hundred-year project. From the very beginning, translation from English to Tibetan has been an integral part of this project because of the need to prepare course materials as well as to facilitate on-site classes and lab activities in the Tibetan language. Our translation process involves not just conveying novel and foreign concepts across cultures but doing so with a scientific language peppered with technical terms that are not readily representable in the target language.

OPEN ACCESS

Edited by:

Robin Nusslock,

Northwestern University,

United States

Reviewed by:

Rachel Jacobs,

Northwestern University,

United States

Marcia Grabowecky,

Northwestern University,

United States

*Correspondence:

Dadul Namgyal

gnamgya@emory.edu

Specialty section:

This article was submitted to

Science and Environmental

Communication,

a section of the journal

Frontiers in Communication

Received: 26 June 2021 Accepted: 19 October 2021

Published: 24 November 2021

Citation:

Samphel T, Namgyal D, Drongbu DT and Khangsar KT (2021) Translation: A

Key Component of a Hundred-

Year Project.

Front. Commun. 6:731354.

doi: 10.3389/fcomm.2021.731354
In addition to the linguistic barriers, cultural and technical ones further complicate the process of communication. A case in point is the concept of life, or correlation versus causation, or the view that consciousness is an emergent property of the brain, where each construct has its corresponding but differing concept in Tibetan Buddhism. When engaging with such existing parallel yet divergent terms or concepts, the translators must strike a delicate balance and avoid forsaking the distinctive characteristics and connotations involved. In this article, the ETSI translation team shares its journey-highlighting the needs felt, challenges faced, and solutions sought. We discuss the translation principles guiding our work and the handling of such scientific features as graphs, acronyms, units, chemical names, and formulas. We hope our work will inspire other similar projects around the globe and encourage them to continue bridging barriers to cross-cultural dialogues, promoting cross-fertilization of knowledge for human flourishing.

Keywords: Dalai Lama, ETSI, science translation, buddhist monastics, science and buddhism, Emory-Tibet science initiative

\section{INTRODUCTION}

The Emory-Tibet Science Initiative (ETSI) is a unique educational project conceived and supported by His Holiness the Dalai Lama, and formally launched in 2008 by Emory University in collaboration with the Library of Tibetan Works and Archives (LTWA), India. An ambitious endeavor anticipated to endure the next hundred years, ETSI has already journeyed through its first three phases of planning, piloting, and implementing systematic and sustainable curriculum to provide modern science education within the Tibetan monastic institutions in India (ETSI, 2019). In 2019, ETSI graduated its first cohort of monastic students-a total of 233 monks from nine monastic academic institutions-who had completed the six-year science curriculum. ETSI has entered the "sustainability" phase focusing on training select groups of monastics in science pedagogy and 
research methodology to assist participating monastic institutions in building an indigenous pool of science teachers and researchers.

In its current form, ETSI focuses its efforts on the scientific disciplines of physics (with some introductory lessons in mathematics and related components), life sciences, neuroscience, and philosophy of science. Studies have shown that "learning academic courses through a foreign language medium may pose conceptual, linguistic and psychological problems" (Sabri et al., 2005). Moreover, since the monastics enter the program with little to no prior knowledge of English language, translation and interpretation of science instructions in Tibetan language has been an integral part of the program from its inception (Gray et al., 2020).

\section{Historical Perspective}

Where does this put ETSI historically in terms of translating modern scientific terminology and concepts into Tibetan? To our knowledge, there had never been a systematic and coordinated effort invested in this direction, at least not at an institutional or state level before 1959, the year when the Chinese Communists invaded and occupied Tibet (Tsering and Gyatso, 2000). Individuals had undertaken sporadic attempts ('Jam dpal chos kyi bstan 'dzin 'phrin las, 2013; Chophel, 2014 and Lopez, 2018), but no documentation of any organized and systematic efforts are to be found. Under the new regime in Tibet, science textbooks written in Tibetan were gradually implemented into curricula, at least at the primary and middle school levels. Such materials primarily addressed topics in general science, life sciences, physics, and chemistry (Ljongs Zhing-lnga'i mnyam bsgrigs, 2006).

ETSI acquired some of these limited classroom materials and has consulted them as references in the program's initial years. However, one prominent gap was the absence of any material in Tibetan on neuroscience. Thus, ETSI's work in neuroscience had to start virtually from ground zero, and to build on a meager basis for the other disciplines. Ironically, among the equally important and relevant fields of science engaged by the program, neuroscience seems to be the one that most readily deals with topics with the greatest potential to foster interdisciplinary discussions and exchanges to which senior monastics would already have much to contribute. Also, considering the program's aspiration to rally contemplative traditions and modern science to promote positive human values towards sustainable global peace and human flourishing, the approach of contemporary neuroscience to the question of consciousness in general, and compassion, empathy, attention, etc. in particular, bring the discipline centerstage. These reasons prompt us to focus this paper on our efforts to translate neuroscience concepts and terminology in capturing the dynamics of our work.

In retrospect, Tibet and Tibetan civilization undertook a major transplantation of Buddhism from India that lasted several centuries before rooting itself deeply into Tibetan culture and flourished locally. The importation of Buddhism started not long after the current written form of Classical Tibetan Language, together with its grammatical rules, was created and introduced during the reign of Tibet's 33rd King, Songtsen Gampo (c. 557-649 AD). The mission was spearheaded by Thonmi Sambhota, who invented the script based on Indian Devanagari scripts after intensive studies in India. Interestingly, around the 8th century, the above enterprise of translating Buddhist canons received royal patronage, which resulted not only in the commissioning of individual translation projects, but also in the founding of general principles and rules governing translation and standardization (Sgra-sbyor bam-gnyis) $^{1}$. These general principles and rules of translation have proven immensely helpful to us. In addition, we use Tibetan medical texts to generate possible ideas and suggestions to render neuroscientific terms and concepts into Tibetan. However, references to brain anatomy in Tibetan Buddhist canons and indigenous writings are tellingly rare (The Dalai Lama, 2017). Even in Tibetan medical texts, neurology is not addressed as a separate area of study. Instead, all references to brain-related concerns are interspersed throughout the chapters in connection with different body systems, pathologies, and treatment (Bdud-rtsi snying-po, 2011). Early on, ETSI team became aware of how differences underpinning cultures, worldviews, concepts, approaches, and systems of classification heavily affected defining a discipline and identifying its components as well as the relationships among them. As a result, ETSI translators were even more wary of borrowing existing Tibetan medical terms and concepts to map them onto a corresponding area in neuroscience, for doing so could potentially hamper doing justice to the purpose and integrity of translation and instead create more misunderstanding, albeit inadvertently.

\section{Translation: Challenges Faced and Methods Adopted}

The work of translating neuroscience materials into Tibetan began from scratch and it involved employing the entire spectrum of translation methods and tools available to the team, which we share here with our readers. First, whenever possible, we aimed for simple direct translations such as in "Dkar-rdzas" for white matter and "Skya-rdzas" for gray matter, where we provided a word-for-word substitution in the same order (Wylie, 1959). Second, when such an orderly arrangement did not fit in the target language, the same wordto-word substitution was pursued with the word order adjusted. For example, "Mdun-gnos klad-shun dkyil-gyi steng-cha" for

\footnotetext{
${ }^{1}$ Sgra-sbyor bam-gnyis. Toh 4347, Dege Tengyur, vol. co (sna-tshogs) page 131b $-160 \mathrm{~B}$. This is a ninth-century lexicon containing specific rules and methods for translating Sanskrit texts into Tibetan and it is accompanied with a full-length glossary of Buddhist terms translated into Tibetan from Sanskrit. Publisher/ Printery: Delhi Karmapae Chodhey, Gyalwae Sungrab Partun Khang; Delhi. 1985. ${ }^{2}$ The transliterations provided in this paper are based on the Wylie system developed initially by Turrell Wylie in 1959 and later extended by a team at the Tibetan and Himalayan Library, co-directed by Nathaniel Garson and David Germano, in a manuscript draft entitled, "THL Extended Wylie Transliteration Scheme", which can be accessed at https://www.thlib.org/reference/transliteration/ \#! essay=/thl/ewts.
} 
"ventral medial prefrontal cortex" and "Mthong-tshor gyi barrim klad-shun" for "secondary visual cortex" are where a direct restoration from the translations would leave us with "prefrontal cortex medial ventral" and "visual secondary cortex", respectively. Sometimes, adding a tag in the translation was called for in the target language when performing a simple direct translation. For example, "Kham-tsig klad-zho" for "amygdala," is where the rear tag "klad-zho", meaning "brain substance", helps put it in the context of brain science. Otherwise, just leaving it as "Kham-tsig," which translates to "almond," its Latin root, could create confusion.

Third, the next option would be to try indirect translation when a simple direct treatment may be impossible or simply not the best option. This method could include several approaches within it. For example, we translated "cell" to "Phra-phung" which literally means "tiny body" or "tiny structure." If we had instead settled for "Shag-khung," which is what the original term conveys, a "small quarter," that might turn out to be an unwise choice, prone to creating unnecessary confusion. Once a term is established, we again return to simple direct translation wherever applicable and possible. This is reflected, for example, in the translation of "nerve cell" and "brain cell" respectively into "Dbang-rtsa phra-phung" and "Klad-pa'i phra-phung" where word-for-word treatment and the same word order are pertinent. Connected with these terms is "neuron" which is translated as "Dbang-rtsa phra-gzugs," a rather wordy choice, but the translation conveys the context along with a reference to a tiny structure through the use of a different word than the one for "cell". These examples illustrate how the team works to retain synonyms in translation, rather than merely lump synonyms from the source language into a single translation in the target language for the sake of expediency. Of particular interest might be how we translated "cell body" as "Phra-phung-lus", not as "Phraphung gi lus" which would be "cell's body or body of a cell", and that is of course not the meaning intended in the source term. Generally, a three syllabled word like "Phra-phung-lus" is a rare occurrence in Tibetan. ${ }^{3}$ We mostly prefer pairing up the syllables. It is also what most of Tibetan linguists would recommend achieving in general writing to avoid leaping over the proper point of break and thus leading to misrepresentation, both in communication as well as in comprehension.

Fourth, sometimes, we use different yet contextually sensitive Tibetan terms for a single scientific word. For example, "nucleus" is translated as "Lte-rdhul" meaning "core particle" for the nucleus of an atom, and "Lte-nying" meaning "coreessence" for the nucleus of cells, and "Tshom-bu" meaning "bundle" for structures like the cochlear nucleus. Likewise, in instances such as the phases of mitosis, we have found it more practical for the purpose of student learning and teaching, to convey the phases with terms that capture the distinctive salient

\footnotetext{
${ }^{3}$ This is an example of loan translation approach. Although it may initially sound strange to a Tibetan ear, it conveys the meaning of the source word clearly and also indicates a foreign source (Heim and Tymowski, 2006).
}

features of each, rather than in strict adherence to the original names. For example, "Stug-ngo", for "prophase", captures the feature of "thickening" of the chromosomes during this phase. Similarly, in connection to the anatomical direction and reference planes of the brain, we did not examine the actual origin of the terms used in the source language. Instead, we settled on using the existing Tibetan terms for spatial orientation and appropriate additions to accurately pinpoint the areas of focus (Eisen, 2012).

Phonetization is another area of interest in any translation project of our scope. A reliable and accurate, albeit a slightly sophisticated, system of phonetization combined with transliteration for Sanskrit terms into Tibetan already existed (Sgra-sbyor bam-gnyis, 1985). This phonetization system was painstakingly developed by our predecessors primarily to retain the purity of tantric mantras, both in sound and meaning. However, this system would be applicable only if the source terms being phonetized and transliterated were already in established Sanskrit or Hindi spellings, or at least in spellings or sounds sharing the same parallel linguistic dynamics as those languages. With English as the source language from which to translate, the above method was not an option for our project. Precisely because of this vacuum regarding a uniformly applicable standard Tibetan phonetization system for languages including English and Chinese, a few loosely defined schools or styles in the Tibetan community exist to represent terms from other languages. A few of these "schools" take a more conformist stand whereby the original sound of the term may be compromised in favor of a more Tibetanized sound or even recognizable Tibetan way of spelling for a foreign name (Ljongs Zhing-lnga'i mnyam bsgrigs, 2006). Yet another school sticks to the term's original sound even if it means settling for a non-Tibetan sound, let alone a non-Tibetan way of spelling the sound. The ETSI translators have opted to stand by this latter style, although without a formal consensus and written rule. Therefore, proper nouns such as Darwin and Mendel would be found as "Dar-win" and "Men-del" respectively in the ETSI textbooks, not as "Tar-win" and "Dhar-win" or "Men-tel" and "Men-dhel", reflective of the other practices. One way of telling the difference in style is to identify whether letter-symbols outside of Tibetan alphabet are used in a text.

Furthermore, not every scientific term is deemed easily translatable or worth the effort in favor of convenience and conveyance. This category includes acronyms such as DNA, RNA, mRNA, etc. The ETSI practice has been to phoneticize these acronyms using the same criteria as above. However, there is a sub-section in this group of acronyms that are better received if expanded in translation rather than just retained in phonetics in Tibetan usage. This relates to acronyms such as MRI, fMRI, EEG, SNRI, etc. which would become too cumbersome and unclear if phoneticized, or at minimum would require tagging with a clue after the phoneticized rendition to hint at the meaning at the cost of residual inconvenience. Associated with this challenge are certain terms such as "lysin" "lysis," or "lysing" where there is 
TABLE 1 | Science textbooks and other bilingual educational materials produced by ETSI to date.

\begin{tabular}{|c|c|c|}
\hline S.No & Subject & Count \\
\hline 1 & Primers: bilingual science textbooks specifically designed and developed & 20 \\
\hline 2 & Supplementary Science Books - bilingual & 2 \\
\hline 3 & English-Tibetan Modern Science Dictionary & 1 \\
\hline 4 & Video Lectures: in-class bilingual videos filmed at the locations & 403 \\
\hline 5 & Distance Learning Videos: bilingual modules on various scientific topics & 112 \\
\hline 5 & ETSI Webinar Recordings & 33 \\
\hline 7 & Presentation Slides: bilingual slides used as teaching and learning tools & 7,768 \\
\hline 8 & Standardized Scientific Terms in Tibetan & 6,300 \\
\hline
\end{tabular}

almost no other option but to resort to phonetics. In doing so, it would be preferable to use a non-Tibetan spelling to give the impression of its being a foreign term at the first sight. That means rendering "lysin" and "lysis" as "Lee-sin" and "Lee-sis" respectively in Tibetan to convey the substance and the biological process of lysing. In this case, the original sound is retained as conveyed in a non-Tibetan spelling reflected in the double slants on top, represented here by "ee." By extension, "lysing," as a verb for a biological process, is rendered as "Leebshig thebs-pa" or "Lee-bshig gtong-wa," with a portion of the phonetics attached to a Tibetan action word, suggestive of the action of either undergoing or causing to undergo lysis (Eisen A., 2018).

Subtle differentiations in scientific concepts such as speed versus velocity, or among power, force, and energy also present a challenge for translation. One option is to coin completely novel allocations, but such neologisms may take too long to be utilized fully. Besides, there already are terms in the Tibetan language that express the general concepts of the words listed in each of the above groups, so efforts to coin new, unfamiliar terms might be superfluous as well as counterproductive. In such situations, we have chosen a different approach of arbitrarily allocating existing synonyms with specific meanings, such as "Mgyogs-tshad" for speed and "Myur-tshad" for velocity, to represent the nuanced notions within the general concept as has been done in English (Hewitt and Samphel, 2010). We hope that overtime these renditions will acquire the specific meanings associated in that context.

Another, formidable challenge for translation is words that represent familiar concepts or notions commonly found in any culture, or discipline around the world. Thus, no one would expect or imagine having to struggle with learning about, say mind, health, life, safety, etc. through totally new and unfamiliar terms. However, there may be very subtle, yet significant differences in the meaning of those words among cultures or disciplines. The ETSI team has its own set of such word groups to tackle and does its best in negotiating the usage in translation. Among these words are "life," "living," "consciousness," and "awareness". Superficially, the words already have their presumed equivalents in the target language and are thus ready to be employed correspondingly. Yet the existing meanings of the words in the two traditions tend to create tension in the actual usage. The
ETSI team continues to use the same familiar terms but warns students of the difference in subtleties during lessons and continues with the narration expecting the terms to be understood from the perspective of their respective traditions. Interestingly, in the context of consciousness, awareness, and the like, one may assume that Buddhism must cover a wide range almost accounting for every imaginable category and have corresponding terms ready. The ETSI team has found that is not always the case. Among all the diverse ways of classifying mental phenomena, Buddhism does not seem to categorize mental phenomena into emotions and cognitions. Hence, it does not have existing terms that distinguish these two broad categories. Textual sources refer to distinguishing features that characterize the categories while explicating and articulating them, but never employ a discrete term for the type. Unsurprisingly, several propositions for formulating these distinctions in Tibetan have emerged in writings or discussions, particularly concerning "emotions" (Khabdha, 2019). However, we invented our own, "Sems-myong," literally translating to "mental experience" or "mental feeling" (Mascaro et al., 2017). "Sems-myong" was chosen over the older terms proposed by others because they already had existing overlapping meanings. The same situation arose for translating the word "cognition." Although we are not completely satisfied with our usage of "Nges-'dzin" as a translation for cognition, we think it is a close translation, but we remain open to better versions.

As the monastic students graduate to the next class every year and with the number of technical terms in translation growing both in volume and usage, the need for a reference material where one can locate words easily and revisit to understand context and meaning is acutely felt. We recently achieved this long-awaited goal of compiling a glossary. In addition, we have published 20 primers for the disciplines covered in ETSI, plus a couple of supplementary books (Table 1).

In translating technical terms, we follow not only guidelines set forth by the great Tibetan translators of the past, but also contemporary practices such as accepting loanword (lezer for laser) and providing loan translation (Phra phung lus for cell body) that are practiced widely in many fields (Heim and Tymowski, 2006). However, the method of 
bringing loan-words into Tibetan as currently practiced by us or other science translators based in India or Tibet is phonetic borrowing rather than transliteration since we don't have a unified standard system of transliterating English to Tibetan-something that the Tibetan translators must develop.

\section{Standardization Process}

We follow a stringent process for standardizing terms to be adopted and used in printed materials. Suggestions for translations of new technical terms encountered and initially refined during informal work by individual translators are welcome from any corner. However, before any word is officially adopted and entered into published works, it must be tabled for discussion and deliberation at the annual translation conference. Experts in different fields of Tibetan studies such as medicine, astrology, Buddhism, poetry, history, and literature are invited to these conferences together with the resident translators of the ETSI and representatives from the LTWA. Conferees deliberate on the new terms and concepts from the different scientific disciplines together with the proposed Tibetan translations. Often, Western experts in the respective fields are also in attendance to shed light on the subtleties and nuances of the terms and concepts. Thus far, 12 annual conferences have been held since 2009, each lasting about a week on average. To date, we have adopted a total of 6,300 new Tibetan terms through this process. As noted earlier, words finalized during these conferences are open to improvement when better choices are formulated and tabled for subsequent meetings and consensus reached. One clear example is the term for "action potential", the Tibetan translation for which underwent a few adjustments before adoption of the current form, "Las-'jug nus-pa" (Eisen et al., 2016). Besides, each final draft translation of a textbook is cross-checked by fellow translators and then sent for vetting, at least for the early textbooks, by Geshe Lhakdor, Director of LTWA.

\section{CONCLUSION}

We are proud to share that our work is impacting our community significantly, particularly within the Tibetan monastic communities and the educated laity. It imports concepts new to the culture and offers corresponding linguistic tools so that the Tibetan language communities can discuss on topics of practical relevance such as science and technology, inventions and innovations, collaboration between science and Buddhism (Khabdha, 2009-21).

${ }^{4}$ Khabdha.org is an online platform for Tibet related news, announcements, and discussions. Browse their website https://www.khabdha.org for modern science related articles, discussions and book announcements. Particularly, search by typing in Tibetan script "tshan rig" for articles and book announcements between 2009 - 2021.
This clearly shows how ETSI is introducing new knowledge and insights into modern science as well as contributing to the vocabulary of Tibetan language to reflect these new frontiers of knowledge. Moreover, and more importantly, these efforts are making a significant contribution in facilitating interdisciplinary discussions as well as encouraging critical, comparative, and analytical writings such as An Anthology of Scientific Knowledge, Ten Reasons Why Monastics Should Study Science, Being Emotional about "Emotions" (Khabdha, 2009-21) ${ }^{4}$, all works of the monastics trained through ETSI or related programs. Such encouraging advances suggest that ETSI is well poised to realize the vision of His Holiness the Dalai Lama to - "(bring) together the best of the Western and Tibetan Buddhist intellectual traditions for their mutual enrichment and for the discovery of new knowledge" (ETSI website, 2021).

Moving forward, we remain committed to and passionate about that shared vision. We will continue our efforts in science translation and vocabulary building; and through that effort, hope to become an effective force for invigorating the ETSI's current new phase of generating new cohorts of monastic research partners and indigenous science teachers. This next phase will undoubtedly make a significant contribution towards making the monastic science education robust, sustainable and productive long term.

We hope our work will inspire other projects of cross-cultural nature around the globe and encourage others to continue overcome barriers to inter-disciplinary and cross-cultural dialogues. We are interested to see this happen particularly in the fields of modern science and compassion, thereby promoting "mutual enrichment" of the participating cultures and leading to "the discovery of new knowledge" conducive to human flourishing.

\section{DATA AVAILABILITY STATEMENT}

The original contributions presented in the study are included in the article/Supplementary Material, further inquiries can be directed to the corresponding author.

\section{AUTHOR CONTRIBUTIONS}

All authors listed have made a substantial, direct, and intellectual contribution to the work and approved it for publication.

\section{ACKNOWLEDGMENTS}

We deeply honor His Holiness the Dalai Lama for his vision and continued support of monastic science education. We are thankful for Geshe Lobsang Tenzin Negi's leadership and the continuous support that we receive from the Center for Contemplative Science and Compassion-based Ethics at Emory University. The translators at the LTWA's Science Department and the LTWA's Director, Geshe Lhakdor have collaborated with us from the beginning and made this journey amply successful. We are indebted to the dedicated members of ETSI faculty for their service and 
guidance, and the participating monasteries for their willingness to take this historic journey with us. We express our gratitude to all the

\section{REFERENCES}

Bdud-rtsi snying-po (2011). Bdud-rtsi Snying-Po Yan-Lag Brgyad-A Gsang-Ba Man-Ngag-Gi-Rgyud Ces Bya-Ba Bzhugs-So. Dharamsala, India: Men-Tsee-Khang.

Chophel, G. (2014). Grains of Gold: Tales of a Cosmopolitan Traveler (University of Chicago Press).

Eisen, A. (2018). Life Sciences Primer IV: Disease, Immunology, and Epidemiology. Editor D. Namgyal, Trans (Atlanta, GA: Emory University).

Eisen, A. (2012). Neuroscience Primer I: Perception and Vision. Editor D. Namgyal, Trans (Atlanta, GA: Emory University).

Eisen, A., Mascaro, J., Hasenkamp, W., and Worthman, C. M. (2016). Neuroscience Primer II: Neurons and Neuronal Activities. Editor D. Namgyal, Trans (Atlanta, GA: Emory University).

ETSI (2019). Emory-tibet Science Initiative Graduation Event: Celebrating a Historic Journey. Atlanta, GA: Emory University. Available at: https://tibet. emory.edu/documents/graduation_etsi_2019_hi_res.pdf.

ETSI website (2021). The Convergence of Science and Inner Values. Available at: https://tibet.emory.edu/about.html.

Gray, K. M., Namgyal, D., Purcell, J., Samphel, T., Sonam, T., Tenzin, K., et al. (2020). Found in Translation: Collaborative Contemplations of Tibetan Buddhism and Western Science. Front. Commun. 4., 2020 Article 76. doi: $10.3389 /$ fcomm. 2019.00076

Heim, M. H., and Tymowski, A. W. (2006). Guidelines for the Translation of Social Science Texts. American Council of Learned Societies.

Hewitt, P. G. (2010). Conceptual Physics: Mechanics. Editor T. Samphel, Trans (Atlanta, GA: Emory University).

'Jam dpal chos kyi bstan 'dzin 'phrin las (2013). 'Jam dpal chos kyi bstan 'dzin 'phrin las (written 1830, published 2013). 'Dzam gling rgyas bshad (Detailed Description of the World). Zi-ling, China: mtsho sngon mi rigs dpe skrun khang. Scanned at Tibetan Buddhist Resource Center, Southwest University of Nationalities, Chengdu, China. Available at http://www.tbrc.org/eBooks/ W1KG16588-I2PD17867-1-386-any.pdf.

Khabdha (2019). Article Titled "Smeng-Wa" by Hortsang Jigme, Generated as many as 17 Comments at the Time of Submitting This Article. Available at: https://www.khabdha.org/?p=97689.

Ljongs Zhing-lnga'i mnyam bsgrigs (2006). Ljongs Zhing-lnga'i mnyam bsgrigs slob gzhi lo dgu'i 'gan babs slob gso'i bslab tshan thad gzhi'i tshod lta'i slob deb skye past participants to the annual translation conferences for their contribution to ETSI science education and beyond.

dngos rig pa lo rim brgyad pa’i smad cha. Zi-ling, China: Mtsho-sngon mi rigs dpe skrun khang.

Lopez, D. S., Jr. (2018). Gedun Chophel-Tibet's Modern Visionary. Boulder, CO: Shambala.

Mascaro, J., Hasenkamp, W., and Worthman, C. M. (2017). Neuroscience Primer III: Emotion and Memory. Editor D. Namgyal, Trans (Atlanta, GA: Emory University).

Sabri, K., Ustunluoglu, E., and Aysel, K. (2005). The Effect of Teaching in Native and Foreign Language on Students' Conceptual Understanding in Science Courses. Asia-Pacific Forum Sci. Learn. Teach. 6 (2). Article 2 Available at: https://www.researchgate.net/publication/26453389_The_effect_of_teaching in_native_and_foreign_language_on_students'_conceptual_understanding in_science_courses.

Sgra-sbyor bam-gnyis (1985). Dege Tengyur. Delhi, India: Delhi Karmapae Chodhey, 131b-160B. vol. co (sna-tshogs).

The Dalai Lama (2017). "Science and Philosophy in the Indian Buddhist Classics," in The Physical World. Editor T. Jinpa (Dharamsala, India: Wisdom), 1.

Tsering, W., and Gyatso, T. (2000). Bod Kyi sGra sGyur Lo rGyus Dang Lo Tsa'a Ba Rim Byon Gyi mZad rNam gSal Ba’i Me Long. China: MiRigs dPe sKrun Khang.

Wylie, T. (1959). A Standard System of Tibetan Transcription. Harv. J. Asiatic Stud. 22, 261-267. doi:10.2307/2718544

Conflict of Interest: The authors declare that the research was conducted in the absence of any commercial or financial relationships that could be construed as a potential conflict of interest.

Publisher's Note: All claims expressed in this article are solely those of the authors and do not necessarily represent those of their affiliated organizations, or those of the publisher, the editors and the reviewers. Any product that may be evaluated in this article, or claim that may be made by its manufacturer, is not guaranteed or endorsed by the publisher.

Copyright (c) 2021 Samphel, Namgyal, Drongbu and Khangsar. This is an openaccess article distributed under the terms of the Creative Commons Attribution License (CC BY). The use, distribution or reproduction in other forums is permitted, provided the original author(s) and the copyright owner(s) are credited and that the original publication in this journal is cited, in accordance with accepted academic practice. No use, distribution or reproduction is permitted which does not comply with these terms. 